\title{
Catalyst-free self-healing bio-based polymers: robust mechanical properties, shape memory, and recyclability
}

Yazhou $\mathrm{Xu}^{\mathrm{ab}}$, Songlin Dai ${ }^{\mathrm{a}}$, Liangwu $\mathrm{Bi}^{\mathrm{ac}}$, Jianxin Jiang ${ }^{\mathrm{b}}$, Haibo Zhang*a and Yuxiang Chen*ac

a.Institute of Chemical Industry of Forest Products, Chinese Academy of Forestry,

National Engineering Laboratory for Biomass Chemical Utilization, Nanjing 210042,

China.

b. College of Materials Science and Technology, Beijing Forestry University, Beijing, 100083, China.

c. Co-Innovation Center of Efficient Processing and Utilization of Forest Resources, Nanjing Forestry University, Nanjing 210037, China. 
Table S1 Compositions, thermal properties and mechanical properties of AESO-EMPA polymers.

\begin{tabular}{|c|c|c|c|c|c|}
\hline \multirow[t]{3}{*}{ Sample } & \multirow[t]{3}{*}{ Gel content } & \multirow[t]{3}{*}{$T_{g}$} & \multirow[t]{3}{*}{$\mathrm{T}_{10 \%}$} & \multirow{3}{*}{$\begin{array}{l}\text { Tensile } \\
\text { strength } \\
(\mathrm{MPa})\end{array}$} & \multirow{3}{*}{$\begin{array}{c}\text { Elongation at } \\
\text { break } \\
(\%)\end{array}$} \\
\hline & & & & & \\
\hline & & & & & \\
\hline AESO-EMPA0. & $89.5 \pm 1.0$ & 40.5 & 291.2 & $2.3 \pm 0.05$ & 410.2 \\
\hline \multicolumn{6}{|l|}{2} \\
\hline AESO-EMPA0. & $90.6 \pm 0.8$ & 48.2 & 293.6 & $6.6 \pm 0.1$ & 256.1 \\
\hline \multicolumn{6}{|l|}{3} \\
\hline AESO-EMPA0. & $92.3 \pm 0.5$ & 55.5 & 298.7 & $9.0 \pm 0.15$ & 150.3 \\
\hline \multicolumn{6}{|l|}{4} \\
\hline AESO-EMPA0. & $93.5 \pm 0.4$ & 62.5 & 301.1 & $15.2 \pm 0.17$ & 88.1 \\
\hline \multicolumn{6}{|l|}{5} \\
\hline AESO-EMPA0. & $95.7 \pm 0.4$ & 68.7 & 305.4 & $21.7 \pm 0.19$ & 33.9 \\
\hline \multicolumn{6}{|l|}{6} \\
\hline AESO-EMPA0. & $97.5 \pm 0.3$ & 76.8 & 308.5 & $25.2 \pm 0.23$ & 17.9 \\
\hline \multicolumn{6}{|l|}{7} \\
\hline AESO-EMPA0. & $98.3 \pm 0.2$ & 82.0 & 311.3 & $29.1 \pm 0.25$ & 8.1 \\
\hline 8 & & & & & \\
\hline
\end{tabular}




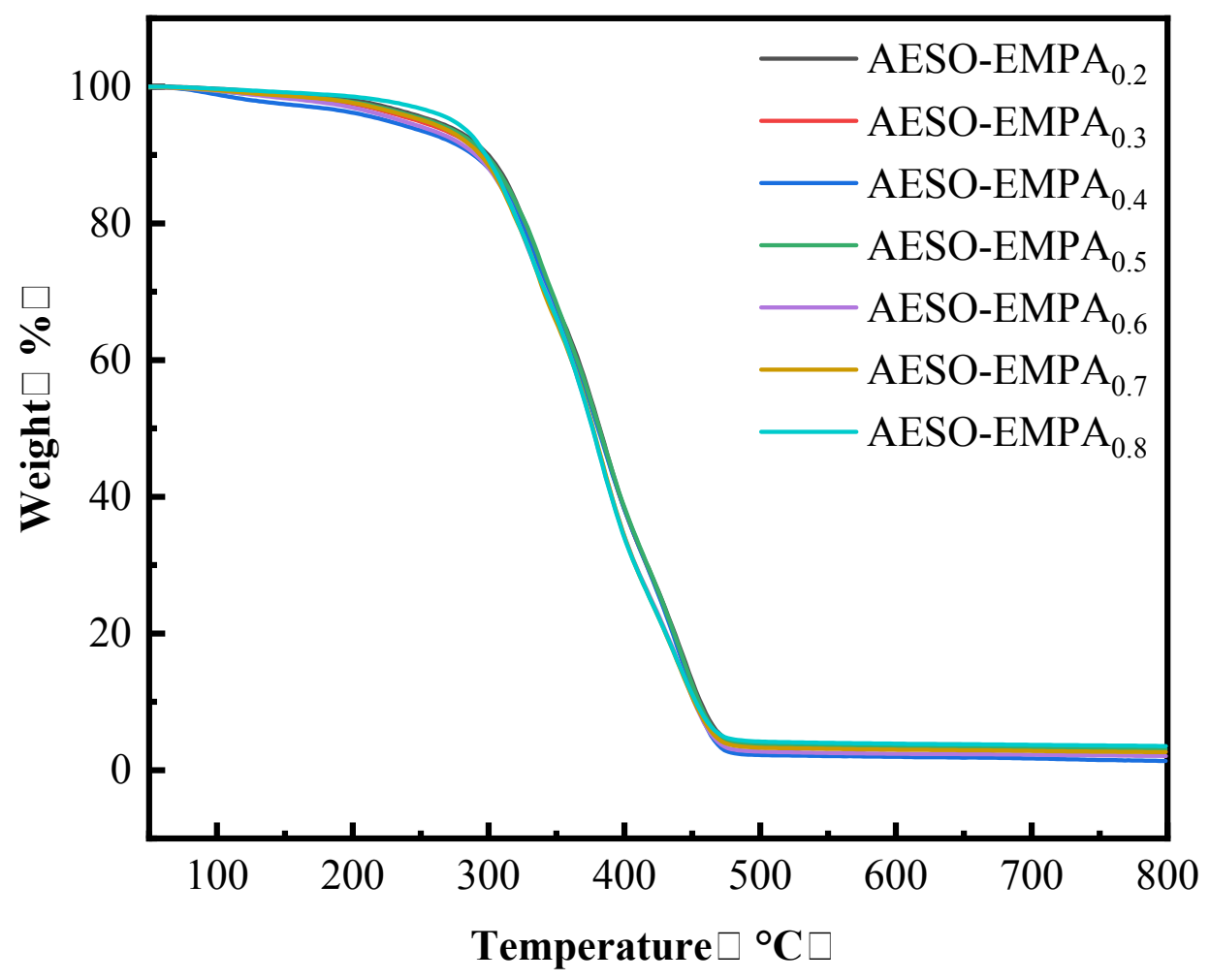

Figure S1.TGA curves of AESO-EMPA polymers. 


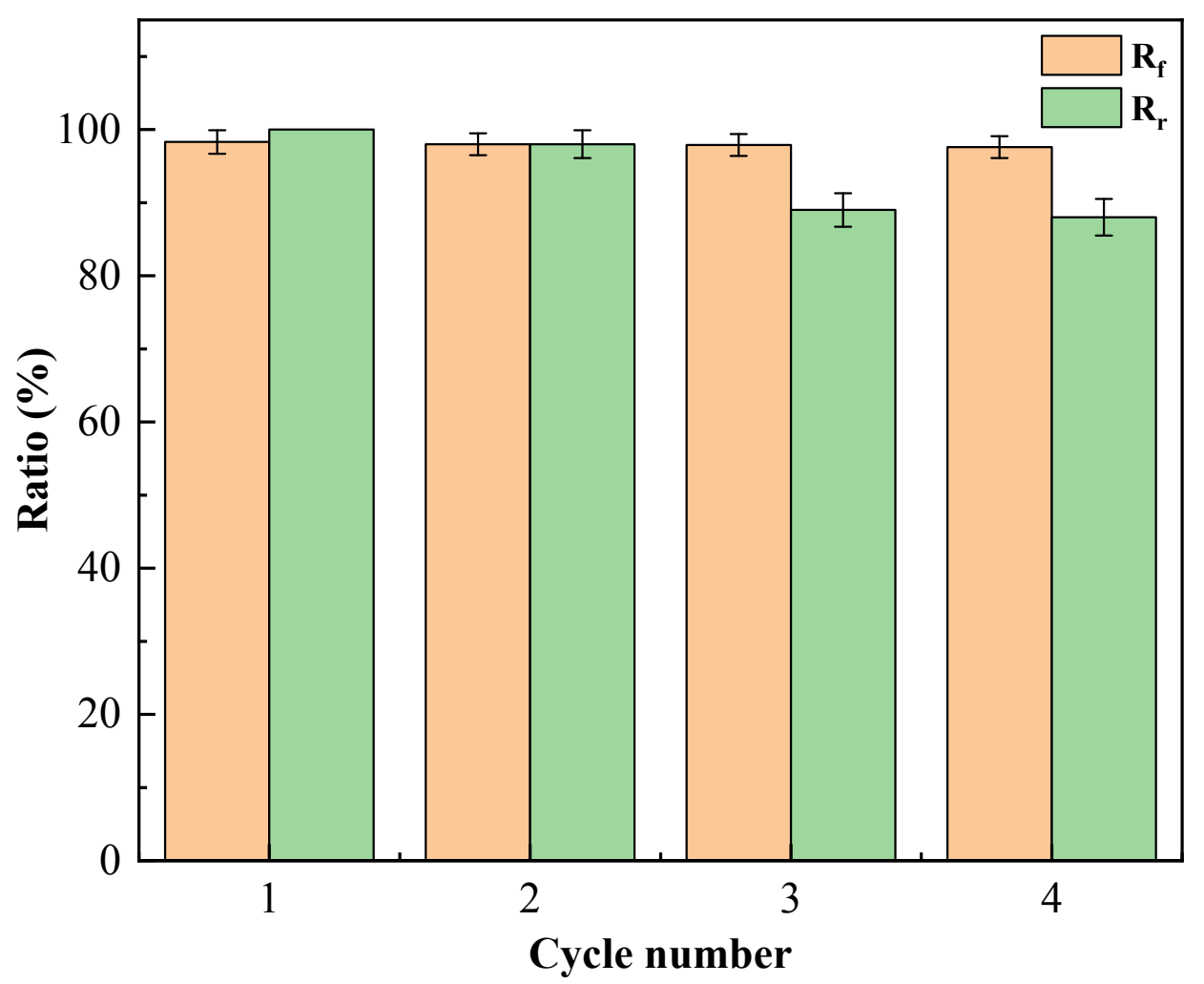

Figure $\mathrm{S} 2$. Shape fixity ratio $\left(\mathrm{R}_{\mathrm{f}}\right)$ and shape recovery ratio $\left(\mathrm{R}_{\mathrm{r}}\right)$ of AESO-EMPA $\mathrm{A}_{0.8}$. 